\title{
MicroRNA-centric measurement improves functional enrichment analysis of co-expressed and differentially expressed microRNA clusters
}

\author{
Su Yeon Lee ${ }^{1}$, Kyung-Ah Sohn ${ }^{1,2}$, Ju Han Kim* \\ From Asia Pacific Bioinformatics Network (APBioNet) Eleventh International Conference on Bioinformatics \\ (InCoB2012) \\ Bangkok, Thailand. 3-5 October 2012
}

\begin{abstract}
Background: Functional annotations are available only for a very small fraction of microRNAs (miRNAs) and very few miRNA target genes are experimentally validated. Therefore, functional analysis of miRNA clusters has typically relied on computational target gene prediction followed by Gene Ontology and/or pathway analysis. These previous methods share the limitation that they do not consider the many-to-many-to-many tri-partite network topology between miRNAs, target genes, and functional annotations. Moreover, the highly false-positive nature of sequence-based target prediction algorithms causes propagation of annotation errors throughout the tri-partite network.

Results: A new conceptual framework is proposed for functional analysis of miRNA clusters, which extends the conventional target gene-centric approaches to a more generalized tri-partite space. Under this framework, we construct miRNA-, target link-, and target gene-centric computational measures incorporating the whole tri-partite network topology. Each of these methods and all their possible combinations are evaluated on publicly available miRNA clusters and with a wide range of variations for miRNA-target gene relations. We find that the miRNAcentric measures outperform others in terms of the average specificity and functional homogeneity of the GO terms significantly enriched for each miRNA cluster.

Conclusions: We propose novel miRNA-centric functional enrichment measures in a conceptual framework that connects the spaces of miRNAs, genes, and GO terms in a unified way. Our comprehensive evaluation result demonstrates that functional enrichment analysis of co-expressed and differentially expressed miRNA clusters can substantially benefit from the proposed miRNA-centric approaches.
\end{abstract}

\section{Background}

MicroRNAs (miRNAs) are short single stranded, noncoding RNAs that regulate protein-coding mRNAs [1-4]. Mature miRNAs cause either target mRNA degradation or translational repression [4] by inducing cleavage or inhibiting translation in the 3'-untranslated regions (UTRs) of the target mRNA $[2,3]$. In spite of the continuous attempts to

\footnotetext{
* Correspondence: juhan@snu.ac.kr

${ }^{1}$ Seoul National University Biomedical Informatics (SNUBI) and Systems Biomedical Informatics Research Center, Div. of Biomedical Informatics, Seoul National University College of Medicine, Seoul 110799, Korea Full list of author information is available at the end of the article
}

identify miRNAs and to elucidate their basic mechanisms of action, little is understood about their biological functions.

Because of the regulatory role of miRNAs [5] and lack of direct functional annotation to miRNAs, current functional enrichment methods for miRNAs rely instead on their target genes' functional annotations [6-8]. If the target genes of a specific miRNA are significantly enriched with a set of Gene Ontology (GO) terms, it is reasonable to infer that the miRNA is also involved in the same GO annotations. As only few experimentally validated targets are available, current methods of target gene's annotation-based
Ciomed Central

() 2012 Lee et al.; licensee BioMed Central Ltd. This is an open access article distributed under the terms of the Creative Commons Attribution License (http://creativecommons.org/licenses/by/2.0), which permits unrestricted use, distribution, and reproduction in any medium, provided the original work is properly cited. 
inference of miRNA function rely on target prediction algorithms such as TargetScan $[9,10]$ and Pictar [11].

Many studies on miRNAs have used this "predicted target-genes' functional annotation-based" miRNA function prediction strategy. Gaidatzis et al. [12] applied a loglikelihood test for functional enrichment analysis for KEGG pathways. Gusev [13] used hypergeometric distributions for GO and pathway-based enrichment analysis. $\mathrm{Xu}$ and Wong [14] applied hypergeometric distribution test to detect significant over-representation of miRNA cluster targets in BioCarta pathways. Similar methods using GO, KEGG and BioCarta pathways were implemented in miRGator [15] and SigTerms [16], applying hypergeometric distributions to evaluate functional enrichment.

The target links from miRNAs to genes, however, show very uneven distributions. So do the links from genes to GO terms. One miRNA may regulate more than several hundreds of targets and one gene may be controlled by many miRNAs [17]. In contrast, the current methods that rely only on the predicted target genes' functional annotations are not powerful enough to capture such variability. For instance, if a certain miRNA targeting hundreds of genes is shared by different miRNA clusters, the clusters' functional annotations may become very similar even though they consist of very different miRNA members, just because they share the 'very bush' one. Another limitation of the current methods is that they treat all target genes equally. One should differently weight genes that are targeted by only one member from those that are targeted by all members of a miRNA cluster. In summary, the current functional enrichment methods for miRNA cluster have limitations of not considering the tri-partite network topologies from miRNAs to genes to functional annotations regarding multiplicity and cooperativity, containing more information than simple target gene counts.

For the purpose of illustration, Figure 1(A) and 1(B) exhibit example cases where the same numbers of miRNAs $(k=5)$ from equal-sized clusters $(k=6)$ are targeting the same numbers of target genes $(k=6)$ from equal number of genes $(k=11)$ that are annotated to a specific GO term, GO:0030282 and GO:0051482, respectively. The numbers of target links between Figure 1(A) and 1(B), however, are differently 8 and 22, respectively. Figure $1(\mathrm{C})$ and 1(D) exhibit cases where the numbers of miRNAs connected to a specific GO term, GO:0015917 and GO:0030851, are differently 6 and 3, respectively, while the numbers of links $(k=6)$ are the same. It is clearly demonstrated that the current approach only based on target gene counts is unable to discern the difference in these targeting relations.

The present study proposes a more generalized conceptual framework to develop and analyze new functional enrichment measures. According to the framework, the traditional "predicted target-genes' functional annotationbased" miRNA function prediction method is regarded as 'target gene-centric' denoted by $\rho$ because it eventually considers only the fraction of the target genes among those that are annotated to a specific GO. Under the proposed framework, we derive 'target link-centric' $(\tau)$ and 'miRNA-centric' $(\mu)$ measures, considering the numbers of links and miRNAs linked to a specific GO term.

Figure 1 illustrates that while the traditional target genecentric $\rho$ measure cannot discern (A) and (B) $(p=$ $0.30325)$ nor $(C)$ and $(D)(p=0.31120)$, the newly proposed $\tau$ and $\mu$ measures successfully discern (A) and (B) (i. e., $p=0.62358$ and $p=0.00956$, respectively) and $(C)$ and (D) (i.e., $p=0.00695$ and $p=0.65253$, respectively). It is clearly demonstrated that different measures calculated from different viewpoints significantly impact the result of functional enrichment analysis of miRNA clusters. We also propose a rank statistic for the purpose of systematic comparison in terms of the average specificity and functional homogeneity of the significantly enriched term for each GO category, Biological Process (BP), Molecular Functions (MF), and Cellular Components (CC). We show that the proposed miRNA-centric measures identify more specific and functionally homogenous sets of GO annotations for miRNA clusters.

\section{Methods}

\section{Dataset: miRNA clusters}

We used publicly available co-expressed and differentially expressed miRNA clusters for comparative evaluation of the proposed methods. For co-expressed miRNA clusters, we obtained the data created by Ruepp et al. [18] that show correlated expression patterns across several human diseases. The data can be downloaded from Ruepp et al. [18] (http://genomebiology.com/content/supplementary/ gb-2010-11-1-r6-s2.xls). Forty three among the 47 clusters having at least one target gene were used in this study. Differentially expressed miRNA sets consisting of up- or down-regulated genes in six solid tumors were also downloaded [19]. MiRNAs down-regulated in colon cancer had no target gene and hence were excluded in the present study. Supplement Tables S1 and S2 in 'Additional file 1' list the $54(=43+(2 \times 6)-1)$ miRNA clusters from the two studies with the associated information.

\section{Creating variations of miRNA-mRNA target pairs for comprehensive evaluation}

Another input of our analysis is the target gene list of each miRNA that will guide the functional enrichment test based on the gene annotations. Considering that only a few experimentally validated miRNA targets are available, we use miRNA-mRNA target pairs obtained from computational target prediction methods. Prediction algorithms generate a relatively high level of false positives [20] and 


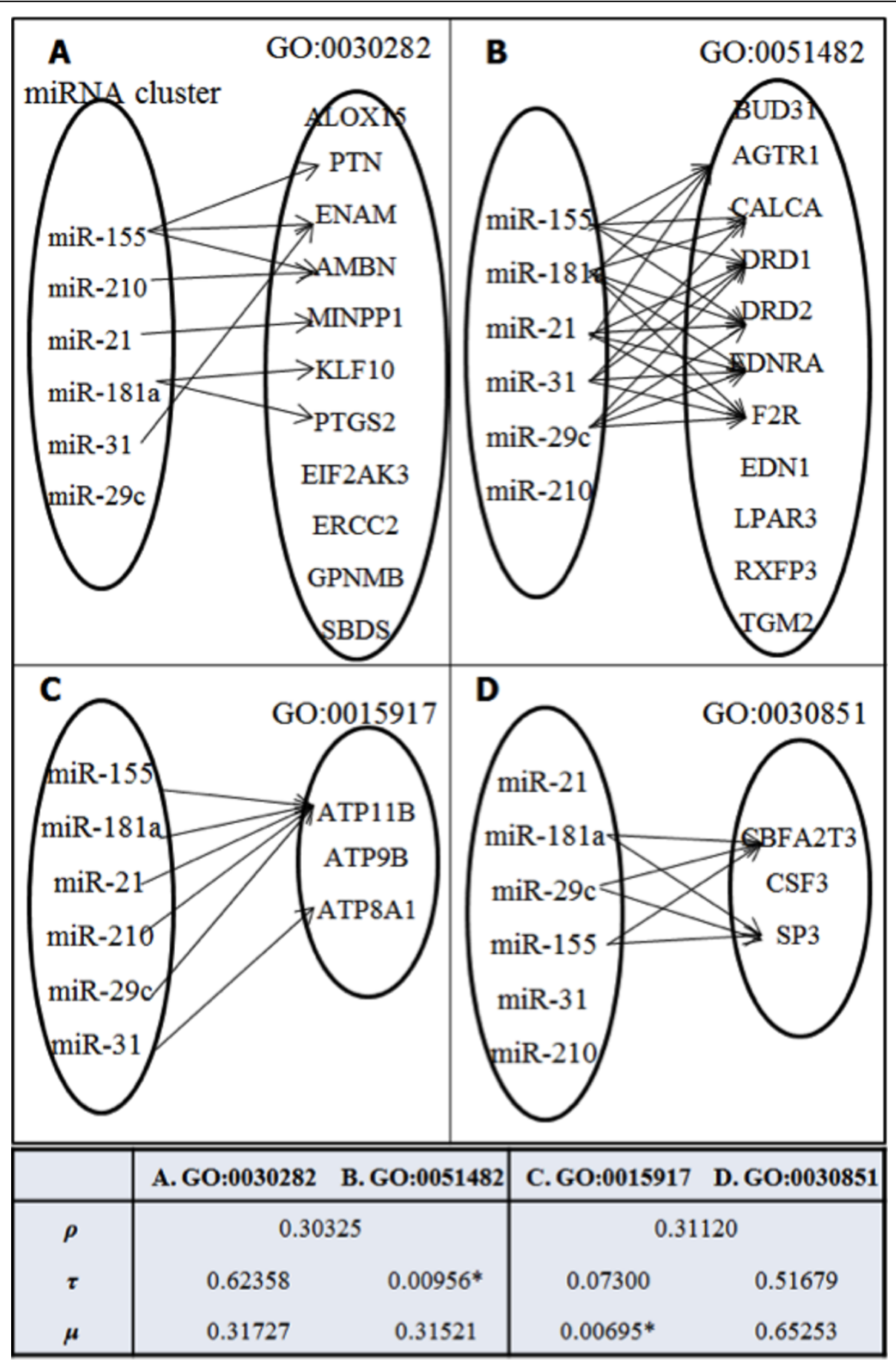

Figure 1 Indiscernibility example. Calculating target gene-centric $(\rho)$ hypergeometric distribution cannot discern the completely different targeting topologies between (A) and (B) and between (C) and (D), resulting the same $p$-values $(p=0.30325$ and 0.31120$)$, respectively. The target link-centric ( $\tau p$-values can discriminate (A) and (B) (i.e., $p=0.62358$ and 0.00956 , respectively) and the miRNA-centric ( $\mu p$-values can discriminate (C) and (D) (i.e., $p=0.00695$ and 0.65253 , respectively). ${ }^{*} p<0.05$, hypergeometric test.

the degree of overlap between predicted targets from different methods is often poor or null [21]. Given the lack of 'gold standard' for miRNA and target gene pairs, we consider a wide range of variations in miRNA-gene pair relations for comprehensive evaluation. We used miRecords [22] and miRGen [23], which are integrated resources of miRNA-target interactions from 11 established target prediction algorithms and from four most 
widely used target prediction programs, respectively. We created 21 variations for predicted target pairs by considering the number of positive voters from the included algorithms by miRecords (Table 1, upper panel) and six variations by applying the four programs of miRGen (Table 1, lower panel). Because most of the evaluation results from these variations were largely comparable, the most representative variation \#6 in Table 1 was used to describe the overall study results in the following sections. Variation \#6 was created by applying the 11 algorithms provided by miRecords, wining more than three positive voters and resulting in 1,569,741 target links from 553 miRNAs to 17,636 genes. As the number of required positive voters is increasing, the numbers of miRNAs, links and genes are decreasing as can be seen in Table 1 .

Target gene-, target relation-, and miRNA-centric calculations of hypergeometric distributions

Now we describe the details of the proposed measures in a proposed conceptual framework. Suppose we want to test the functional enrichment of a miRNA cluster with respect to a specific GO term (or annotation). In most previous approaches, one first constructs a corresponding target gene cluster consisting of all the genes targeted by at least one member in the miRNA cluster. Then the numbers of target genes annotated $\left(\rho_{i}\right)$ and not annotated $\left(\rho_{j}\right)$ by the GO term are used in the two by two contingency table along with the numbers of genes not in the target cluster and are either annotated $\left(\rho_{k}\right)$ or not annotated $\left(\rho_{l}\right)$ with the term, as shown in Figure 2(B). Functional enrichment is tested from this contingency table using a hypergeometric distribution. These traditional target gene-centric $(\rho)$ methods are limited in that they consider only the fraction of target genes connected to a specific annotation for each annotation [12-14], as already illustrated in Figure 1. To this rather confusing problem, the diagram and contingency tables in Figure 2 provide a conceptual framework to understand and correctly design new functional enrichment measures. The diagram of miRNA, gene and

Table 1 Variation for predicted miRNA-gene target pairs

\begin{tabular}{|c|c|c|c|c|}
\hline \multirow[t]{2}{*}{ Index } & \multirow[t]{2}{*}{ No. of algorithms showing positive voting } & \multicolumn{2}{|c|}{ Numbers of } & \multirow[b]{2}{*}{ Genes } \\
\hline & & miRNAs & Target links & \\
\hline \multicolumn{5}{|c|}{ miRecords (Xiao et al., 2009) } \\
\hline$\# 1$ & 3 algorithms & 553 & $1,234,390$ & 17,602 \\
\hline$\# 2$ & 4 & 535 & 272,505 & 15,278 \\
\hline \#3 & 5 & 407 & 53,041 & 9,747 \\
\hline \#4 & 6 & 159 & 9,691 & 2,783 \\
\hline$\# 5$ & 7 & 29 & 68 & 66 \\
\hline \#6 & $3 \sim 11$ & 553 & $1,569,741$ & 17,636 \\
\hline \#7 & $4 \sim 11$ & 535 & 335,351 & 15,422 \\
\hline \#8 & $5 \sim 11$ & 408 & 62,846 & 9,851 \\
\hline \#9 & $6 \sim 11$ & 159 & 9,805 & 2,816 \\
\hline$\# 10$ & $7 \sim 11$ & 40 & 114 & 104 \\
\hline$\# 11$ & $3 \sim 11$ including DIANA-microT & 0 & 0 & 0 \\
\hline$\# 12$ & $3 \sim 11$ including Microinspector & 56 & 184 & 160 \\
\hline$\# 13$ & $3 \sim 11$ including miRanda & 552 & $1,416,379$ & 17,584 \\
\hline$\# 14$ & $3 \sim 11$ including mirtarget2 & 530 & 184,544 & 13,841 \\
\hline \#15 & $3 \sim 11$ including miTarget & 0 & 0 & 0 \\
\hline \#16 & $3 \sim 11$ including NBmiRTar & 42 & 201 & 172 \\
\hline$\# 17$ & $3 \sim 11$ including PicTar & 163 & 64,658 & 6,515 \\
\hline \#18 & $3 \sim 11$ including pita & 551 & $1,559,586$ & 16,676 \\
\hline \#19 & $3 \sim 11$ including rna22 & 54 & 232 & 197 \\
\hline \#20 & $3 \sim 11$ including rnahybrid & 552 & $1,548,423$ & 17,630 \\
\hline \#21 & $3 \sim 11$ including TargetScan & 412 & 343,190 & 16,127 \\
\hline \multicolumn{5}{|c|}{ miRGen [23] } \\
\hline$\# 22$ & DIANA-microT & 175 & 1,816 & 1,206 \\
\hline$\# 23$ & miRanda (microrna.org) & 469 & 430,878 & 16,699 \\
\hline$\# 24$ & miRanda (miRBase) & 156 & 38,821 & 5,444 \\
\hline$\# 25$ & PicTar (4-way) & 177 & 68,100 & 6,391 \\
\hline \#26 & PicTar (5-way) & 128 & 22,028 & 2,433 \\
\hline$\# 27$ & TargetScans & 237 & 75,044 & 7,546 \\
\hline
\end{tabular}




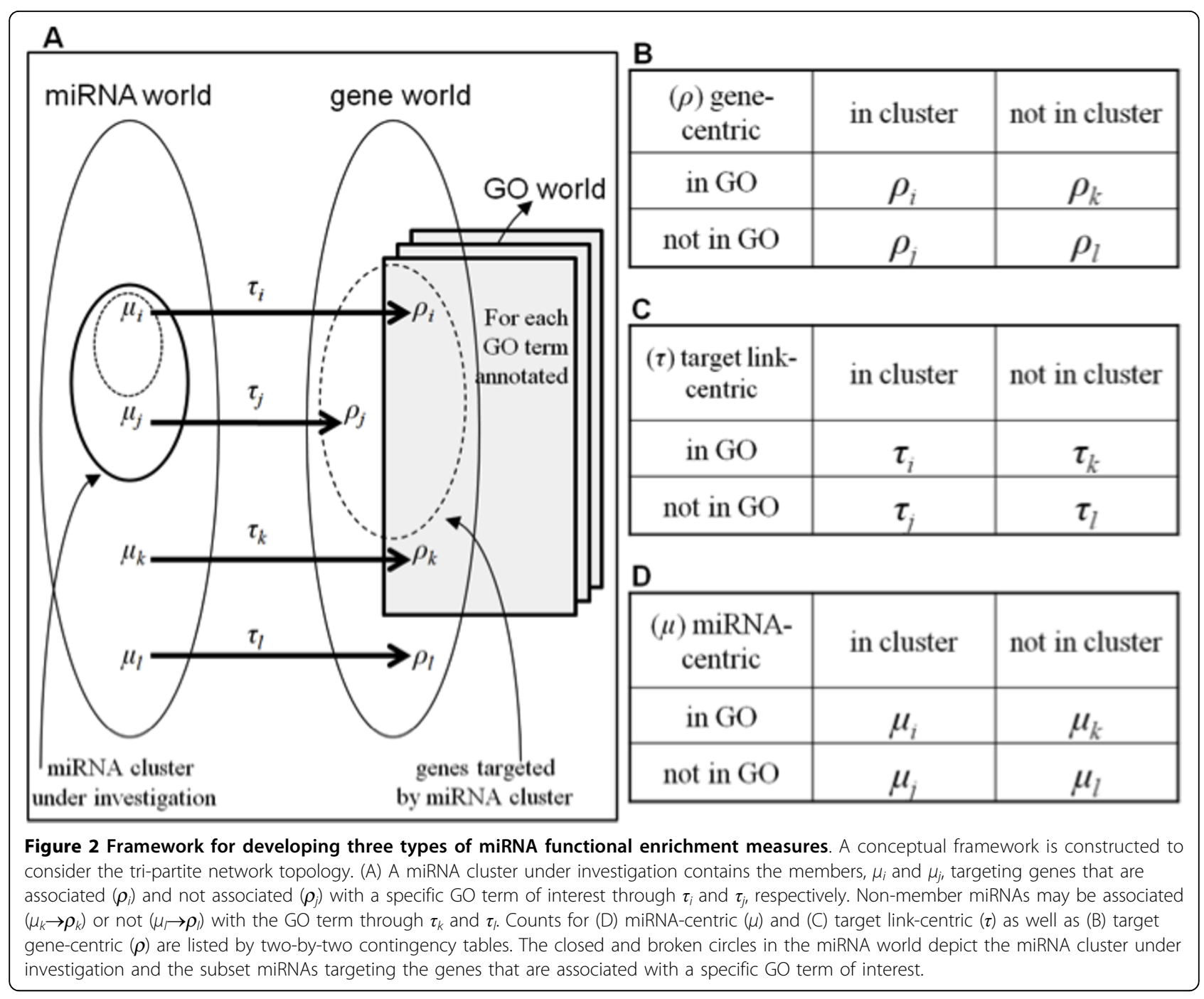

annotation worlds in Figure 2(A) depicts the tri-partite network topology between the three worlds such that one can drive the quartet numbers to create contingency tables for miRNA-centric $(\tau)$ and target link-centric $(\mu)$ as well as for the target gene-centric $(\rho)$ measures (Figure 2(B) (D)).

Under this conceptual framework in Figure 2, subscripts $i$ and $k$ represent positive and subscripts $j$ and $l$ negative connections to the GO term. Subscripts $i$ and $j$ represents connections from inside of and $k$ and $l$ from outside of the targeting miRNA or target gene clusters. The traditional $\rho_{i}$ and $\rho_{i}$, for example, correspond to the sets of target genes that are annotated $\left(\rho_{i}\right)$ and not annotated $\left(\rho_{j}\right)$ to a specific GO term. $\rho_{k}$ and $\rho_{l}$ denote non-targeted genes that are annotated $\left(\rho_{k}\right)$ and not annotated $\left(\rho_{l}\right)$ to the GO term. We can develop a miRNA-centric measure in the conceptualized three framework in a consistent way. We define $\mu_{i}$ and $\mu_{j}$ as the miRNAs in the cluster whose target genes are annotated $\left(\mu_{i}\right)$ and not annotated $\left(\mu_{j}\right)$ to the GO term. As in the case of a gene-centric measure, $\mu_{k}$ and $\mu_{l}$ correspond to miRNAs outside of the cluster whose target genes are annotated $\left(\mu_{k}\right)$ and not annotated $\left(\mu_{k}\right)$ to the GO term. Similarly, for a target link-centric measure, we define $\tau_{i}$ and $\tau_{j}$ as the target links connecting members of the miRNA cluster in $\mu_{i}$ and in $\mu_{j}$, respectively, to genes that are connected $\left(\rho_{i}\right)$ and not connected $\left(\rho_{j}\right)$ to a specific GO term. Remaining miRNAs outside the cluster, $\mu_{k}$ and $\mu_{l}$, target genes through $\tau_{k}$ and $\tau_{l}$ that are headed to genes that are connected $\left(\rho_{k}\right)$ and not connected $\left(\rho_{l}\right)$ to the GO term.

To formally define the three measures, let $\rho$, $\tau$, and $\mu$ be the random variables that represent the number of target genes, target links, miRNAs, respectively, which are linked to a specific GO term as explained above. The following three equations, (1), (2), and (3), describe the hypergeometric distributions of $\rho, \tau$, and $\mu$, respectively. 


$$
\begin{gathered}
\operatorname{probability}\left(\rho=\rho_{i}\right)=\frac{\left(\begin{array}{c}
\rho_{i}+\rho_{k} \\
\rho_{i}
\end{array}\right)\left(\begin{array}{c}
\rho_{j}+\rho_{l} \\
\rho_{j}
\end{array}\right)}{\left(\begin{array}{c}
\rho_{i}+\rho_{j}+\rho_{k}+\rho_{l} \\
\rho_{i}+\rho_{j}
\end{array}\right)} \\
\operatorname{probability}\left(\tau=\tau_{i}\right)=\frac{\left(\begin{array}{c}
\tau_{i}+\tau_{k} \\
\tau_{i}
\end{array}\right)\left(\begin{array}{c}
\tau_{j}+\tau_{l} \\
\tau_{j}
\end{array}\right)}{\left(\begin{array}{c}
\tau_{i}+\tau_{j}+\tau_{k}+\tau_{l} \\
\tau_{i}+\tau_{j}
\end{array}\right)} \\
\operatorname{probability}\left(\mu=\mu_{i}\right)=\frac{\left(\begin{array}{c}
\mu_{i}+\mu_{k} \\
\mu_{i}
\end{array}\right)\left(\begin{array}{c}
\mu_{j}+\mu_{l} \\
\mu_{j}
\end{array}\right)}{\left(\begin{array}{c}
\mu_{i}+\mu_{j}+\mu_{k}+\mu_{l} \\
\mu_{i}+\mu_{j}
\end{array}\right)}
\end{gathered}
$$

Note that for notational convenience, we now used $\rho_{a}$ $\tau_{a} \mu_{a}$ for $a \in\{i, j, k, l\}$, instead of $\left|\rho_{a}\right|$, etc., to represent the number of members in the corresponding set by abuse of notation. The $p$-value for the enrichment test from hypergeometric distribution of the random variable $\rho$ is calculated from the cumulative probability of observing at least $\rho_{i}$ out of $\rho_{i}+\rho_{j}$ times. Accordingly, the $p$-value from each of the three measures can be defined as follows;

$$
\begin{aligned}
& p-\text { value }_{\rho}=\operatorname{probability}\left(\rho \geq \rho_{i}\right) \\
& p-\text { value }_{\tau}=\operatorname{probability}\left(\tau \geq \tau_{i}\right) \\
& p-\text { value }_{\mu}=\operatorname{probability}\left(\mu \geq \mu_{i}\right)
\end{aligned}
$$

These probabilities are computed using the phyper and dhyper functions in R 'stats' package.

\section{Combining P-values}

For the purpose of comprehensive evaluation, we create all possible combinations of the three measures and tested each of those at all GO categories and using different miRNA-target gene pair sets. Figure 3 illustrates steps of combining the three types of hypergeometric distributions for $\rho, \tau$ and $\mu$. For each of the 54 miRNA clusters, of the 27 variations for miRNA-target gene pairs, of the three GO categories, and of annotations (or GO terms), three $p$-values, $p_{\rho}, p_{\tau}$ and $p_{\mu}$, are first computed. Then, we generate 4 combined $p$-values by using Fisher's combined $p$-value method [24].

$p_{\rho, \tau}$ : combined $\mathrm{p}$ - value of $p_{\rho}$ and $p_{\tau}$

$p_{\rho, \mu}:$ combined $\mathrm{p}$ - value of $p_{\rho}$ and $p_{\mu}$

$p_{\tau, \mu}:$ combined $\mathrm{p}$ - value of $p_{\tau}$ and $p_{\mu}$

$p_{\rho, \tau, \mu}$ : combined $\mathrm{p}$ - value of $p_{\rho}, p_{\tau}$ and $p_{\mu}$

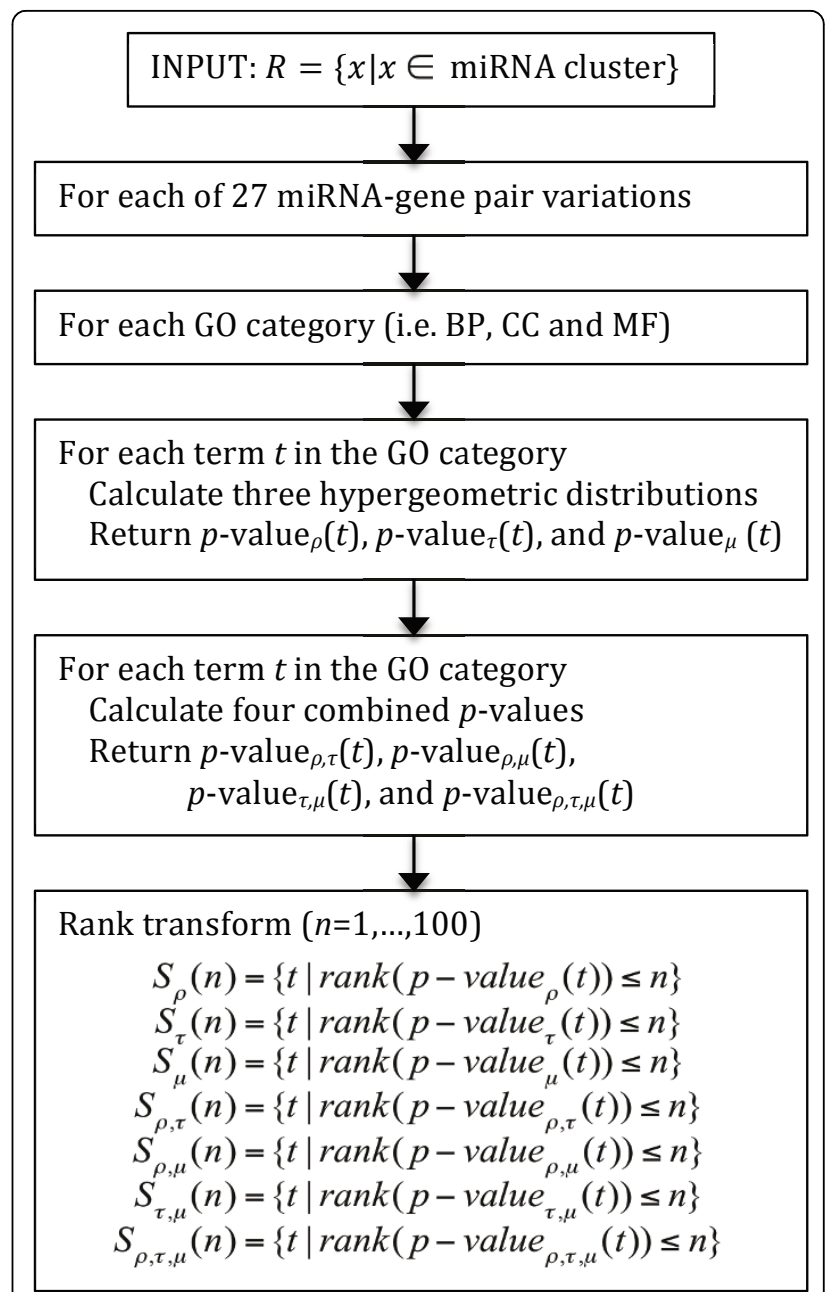

Figure 3 Steps for combining three types of $\boldsymbol{p}$-values. For a selected GO category and a miRNA-gene target-pair variation, for each GO term, three $p$-values are computed for $\rho, \tau$, and $\mu$, and then rank normalized. $S_{\rho}(n)$ denotes the set of $\mathrm{GO}$ terms whose $p$ values' ranks in the $\rho$ hypergeometric distribution are less than or equal to $n$. By applying set operations, four combinations of $S_{\rho}(n)$, $S_{\tau}(n), S_{\mu}(n)$ are created for further evaluation.

We briefly describe how Fisher's combined $p$-value method can be applied to our proposed measures. Under the null hypothesis of no significant enrichment, the individual $p$-value for the random variable $\rho, \tau$, or $\mu$ follows the uniform distribution on $0[1]$. Then the distribution of

$$
Y=-\ln (p-\text { value })
$$

is chi-squared with one degree of freedom. We have three $p$-values from $\rho$, and $\mu$ hypergeometric distributions,

$$
p_{\rho}, p_{\tau} \text { and } p_{\mu,}
$$


and thus we define

$$
Y_{\rho}=-\ln \left(p_{\rho}\right), Y_{\tau}=-\ln \left(p_{\tau}\right), \text { and } Y_{\mu}=-\ln \left(p_{\mu}\right)
$$

Each of the random variables $Y_{\rho}, Y_{\tau}$, and $Y_{\mu}$ is under the chi-squared distribution with one degree of freedom. The final four sums of $W$ are then defined as follows:

$$
\begin{gathered}
W_{1}=Y_{\rho}+Y_{\tau} \\
W_{2}=Y_{\rho}+Y_{\mu} \\
W_{3}=Y_{\tau}+Y_{\mu} \\
W_{4}=Y_{\rho}+Y_{\tau}+Y_{\mu}
\end{gathered}
$$

The random variables $W_{1}, \ldots, W_{4}$ follow chi-squared distribution with degrees of freedom $2,2,2$, and 3 , respectively. These random variables are used to produce the combined 'overall' $p$-values. To calculate these $p$-values, we applied fisherSum function in R 'MADAM' package [25].

The underlying distribution of $\mathrm{p}$-values from each method can be different due to the different characteristics of the measure. To take into account this heterogeneity in the distribution of $p$-values, we rank-normalized $p$-values for each GO category as shown in the last step of Figure 3. Specifically, we construct the set $S_{\theta}(n)$ of top $n$ significant GO terms having the smallest $p$-values for each measure $\theta \in\{\rho, \tau, \mu\}$. Four additional sets of $S_{\rho, \tau}(n)$, $S_{\rho, \mu}(n), S_{\tau, \mu}(n)$, and $S_{\rho, \tau, \mu}(n)$ for the combined measures are also created and used for further evaluation.

\section{Evaluation measures}

Average specificities and functional homogeneity index (or semantic similarity density) of the rank normalized term sets $S_{\theta}(n)$ for each measure $\theta \in\{\rho, \tau, \mu,(\rho, \tau),(\rho, \mu)$, $(\tau, \mu),(\rho, \tau, \mu)\}$ are computed for performance comparison. This is based on the general assumption that for a specific set of GO terms identified by each measure, the more functionally homogenous the set is, the more reliable the measure is. In addition, higher specificities are more desirable because it is more informative to have more specific terms than more general terms in the functional analysis of clusters.

Many studies have shown that Information Content (IC) can quantify the specificity of a cluster [26,27]. IC measure is based on the fact that less frequently used terms are more specific. The IC of a GO term $t$ is defined as follows:

$$
I C(t)=-\log \left(\frac{\text { freq }(t)}{\text { freq }(\text { root })}\right)
$$

where root represents the root term for each GO category. freq $(t)$ is defined as follows;

$$
\text { freq }(t)=n(\text { annotate }(t))+\sum_{c \in \text { children }(t)} n(\text { annotate }(c))
$$

where children $(t)$ returns the list of child terms of term $t$. Thus $t$ becomes a parent term of all members of children $t$ ), either directly or indirectly. The functions annotate $(t)$ and $n(G)$ return the list of genes that are annotated to GO term $t$ and the number of the genes in the gene list $G$, respectively. We use the average IC value of the given term set as a performance measure to compare the specificity.

For functional homogeneity index (or semantic similarity density), we choose a widely used Resnik's measure of semantic similarity [28]. The semantic similarity between two terms is defined as the IC of the lowest common ancestor (LCA) of the two terms and hence is obtained by:

$$
S_{\text {Resnik }}\left(t_{A}, t_{B}\right)=I C\left(L C A\left(t_{A}, t_{B}\right)\right)
$$

As an evaluation measure, the average of all pairwise term-to-term Resnik's similarities was applied for $S_{\theta}(n)$ for each measure $\theta \in\{\rho, \tau, \mu,(\rho, \tau),(\rho, \mu),(\tau, \mu),(\rho, \tau, \mu)\}$ and defined as semantic similarity density of the set.

GO terms and associated gene sets were downloaded from http://www.geneontology.org/gene-associations/ gene_association.goa_human.gz. We excluded GO associations having ND (No biological data) or NR (Not Recorded) evidence codes.

\section{Results}

\section{Average specificity and functional homogeneity index distributions}

Figure 4 shows the distributions of average IC values and functional homogeneity index for GO BP terms with $p$ values in top $n=100$ ranks in the 'breast/up-regulated miRNA cluster' from Volinia et al. [19] (Supplementary Table S2 in 'Additional file 1'). Most of the highest average IC and functional homogeneity values were obtained by miRNA-centric $\mu$ measures throughout the evaluations (see Supplement Fig. S1 series in 'Additional file 1') including the specific example shown in Figure 4. Because of the small numbers of miRNA members and target genes, target variations \#5, \#10, \#11, \#15, and \#16 in Table 1 had no significant GO terms. Evaluation showed that miRNA-centric $\mu$ measure exhibited the best specificity and homogeneity except only for the target variations \#12, \#19 and \#22. The very small numbers of miRNAs (i.e., $m=56,54,175$, respectively) and target genes (i.e., $m=160,197,1206$, respectively) from the very strict thresholds may explain the results. These findings are also consistent throughout the evaluation study regardless of different $\mathrm{GO}$ categories.

\section{Performance comparison with a varying parameter setting}

Figure 5(A) and 5(B) shows the distributions of the average $\mathrm{IC}$ values and functional homogeneity values 

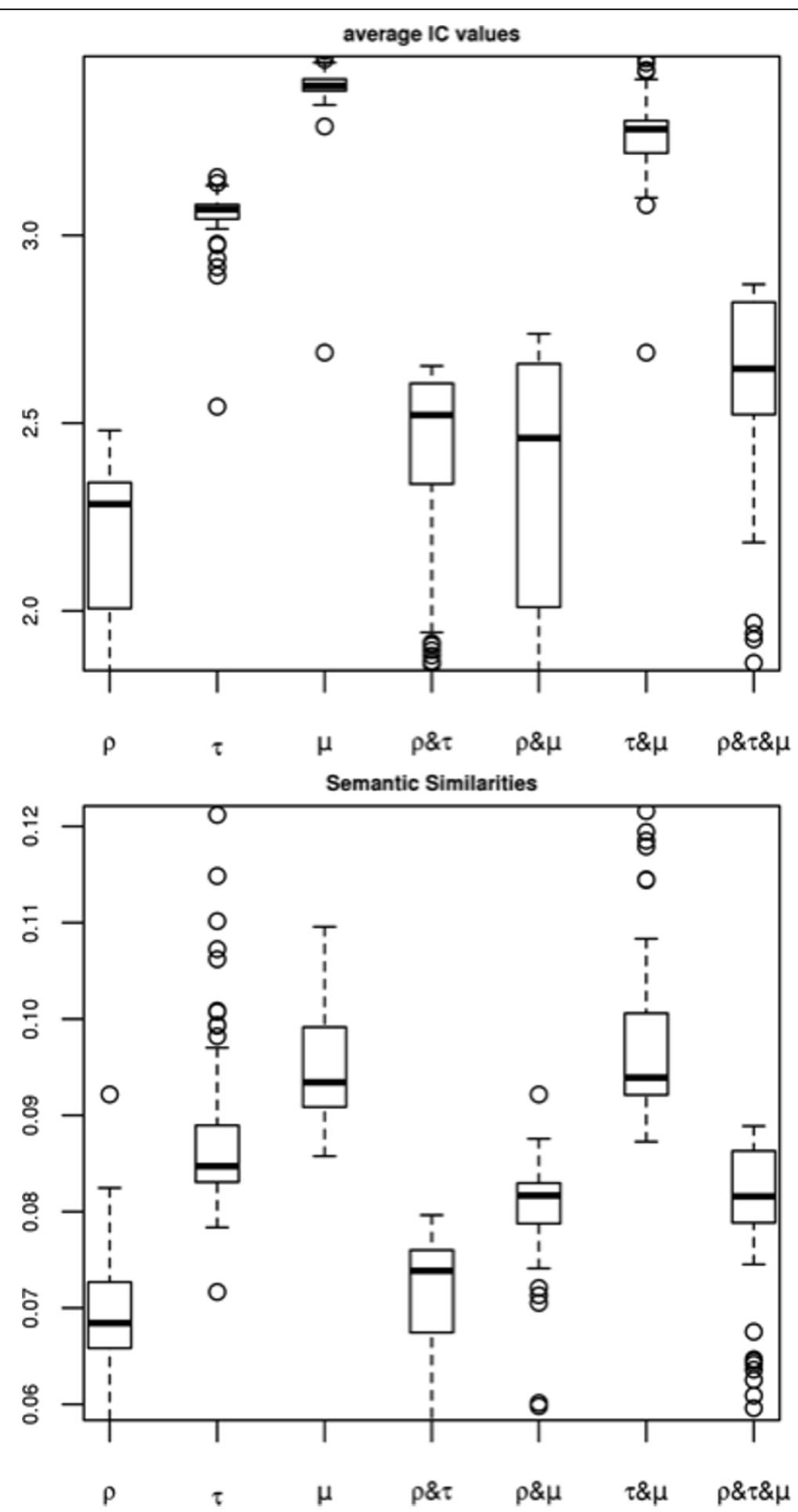

Figure 4 Evaluation of functional enrichment measures and their combinations. Distributions of (A) functional homogeneity index (or average IC value) and (B) semantic similarity (or average all pair-wise Resnik's similarity) are exhibited for significantly enriched GO BP terms in the 'breast/up-regulated miRNA cluster' from Volinia et al. [19](see index 1 in Supplement Table S2) by applying target variation \#6 in Table 1. MicroRNA-centric measure $(\mu)$ outperforms the traditional target gene-centric measure $(\rho)$ and others. 


\section{(A)}
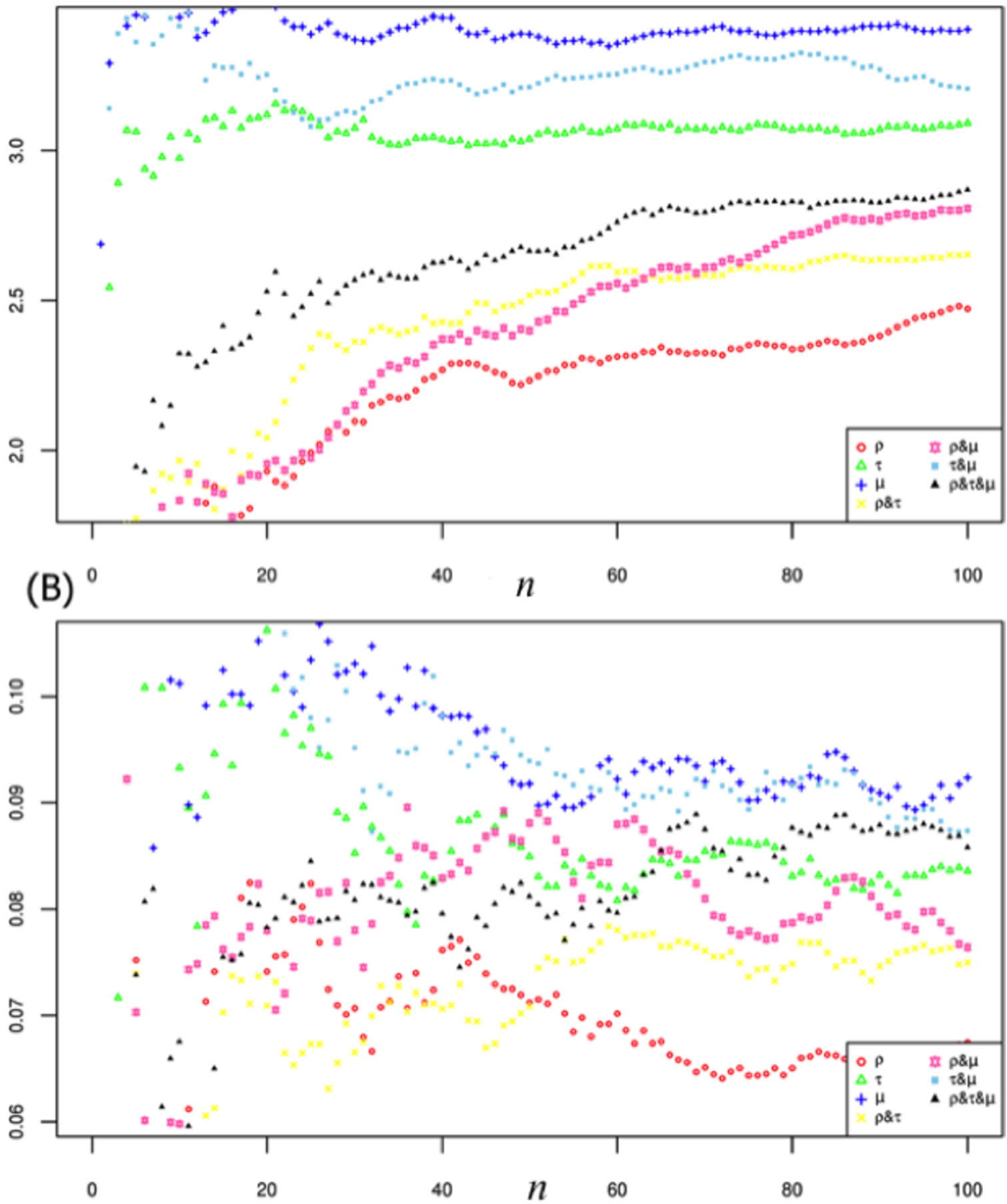

Figure 5 Evaluation of functional homogeneity and semantic similarity densities across different thresholds. Average (A) information content and (B) all pair-wise semantic similarity values are plotted with increasing numbers of rank normalized GO terms $n$ (see Fig. 3) for "breast/up-regulated miRNA cluster" from Volinia et al. [19] (index 1 in Supplementary Table S1 in 'Additional file 1') by applying target variation \#6 in Table 1, GO BP category. Measures containing miRNA-centric $\mu$ (in blue) like $(\rho, \mu)$ (in pink) and $(\tau, \mu)$ (in sky blue) consistently outperform traditional gene-centric $\rho$ (in red) measures at all levels. 
with increasing numbers of rank normalized GO terms $n$ (see Figure 3), as an example for "breast/up-regulated miRNA cluster" from Volinia et al. [19] (index 1 in Supplementary Table S1 in 'Additional file 1') by applying target variation \#6 in Table 1, GO BP category. Measures containing miRNA-centric $\mu$ (in blue cross) like $(\rho, \mu)$ and $(\tau, \mu)$ consistently outperformed traditional gene-centric $\rho$ (in red circle) at all threshold levels of $n$. Figure 6 demonstrates the distribution of $p$-values for all GO BP terms annotated to the miRNA clusters from the dataset of Volinia et al. [19]. Although the interpretation about the $\mathrm{p}$-value distribution is generally tricky and needs to be done carefully, it seems that the $p$-value distribution for miRNA-centric $\mu$ (in green) shows overall better discriminant power than target link-centric $\tau$ (in blue) and traditional gene-centric $\rho$ (in red) methods.

\section{Examples showing complementary properties}

Examples of GO terms determined to be statistically significant by miRNA-centric $\mu$ but not by traditional genecentric $\rho$ method are listed in the upper part of Table 2 . Gusev [13] correctly pointed out that it was common for top ranked GO terms to be targeted by every member of the corresponding miRNA cluster. Those that are targeted by all six miRNA members (i.e., $\mu_{i}=6$ ) shown in the upper part of Table 2, however, are not statistically significant $(p>0.05)$ and show poor ranks $(>290)$ by $\rho$ method. But $\mu$ method shows statistical significances $(p<$
$0.05)$ with high ranks $(<35)$ (Table 2$)$. In contrast, those that are targeted by all six miRNA members shown in the middle part of Table 2 show very strong statistical significance $(p<0.001)$ by $\rho$ method. The very low $\mu_{k}$ to $\mu_{l}$ ratios (i.e., about 50:1) in the middle part compared to those in the upper part (i.e., about 1:1) of Table 2 clearly explain the poor $p$-values and ranks $(>2500)$ by $\mu$ method. Therefore, Gusev's correct intuition can further be formally analyzed by introducing miRNA-centric $\mu$ method. It is demonstrated that our new measure considering $\mu$ complements some drawbacks of the traditional gene-centric $\rho$ measure.

The GO terms in the lower part of Table 2 are annotated only to two to five among six mRNA members such that they are far from statistical significance by $\rho$ calculations. The $p$-values by $\mu$ method, however, are even more statistically significant. Complement activation (GO:0006956) in GO BP category was rejected by the traditional $\rho$ method $(p=0.42)$ but accepted by miRNAcentric $\mu$ method $(p>0.001)$ with ranks of 1251 and 1 , respectively. Complement activation indeed has long been well recognized in breast cancer $[29,30]$. At least four well-known breast cancer genes including SMAD2, SMAD4, TGFB3 and TGFBR3 are involved in palate development. There are many studies reporting that regulation of growth hormone secretion (GO:0060123) is indeed associated with breast cancer [31-33]. For the GO term, negative regulation of activin receptor signaling pathway (GO:0032926), many studies reported that

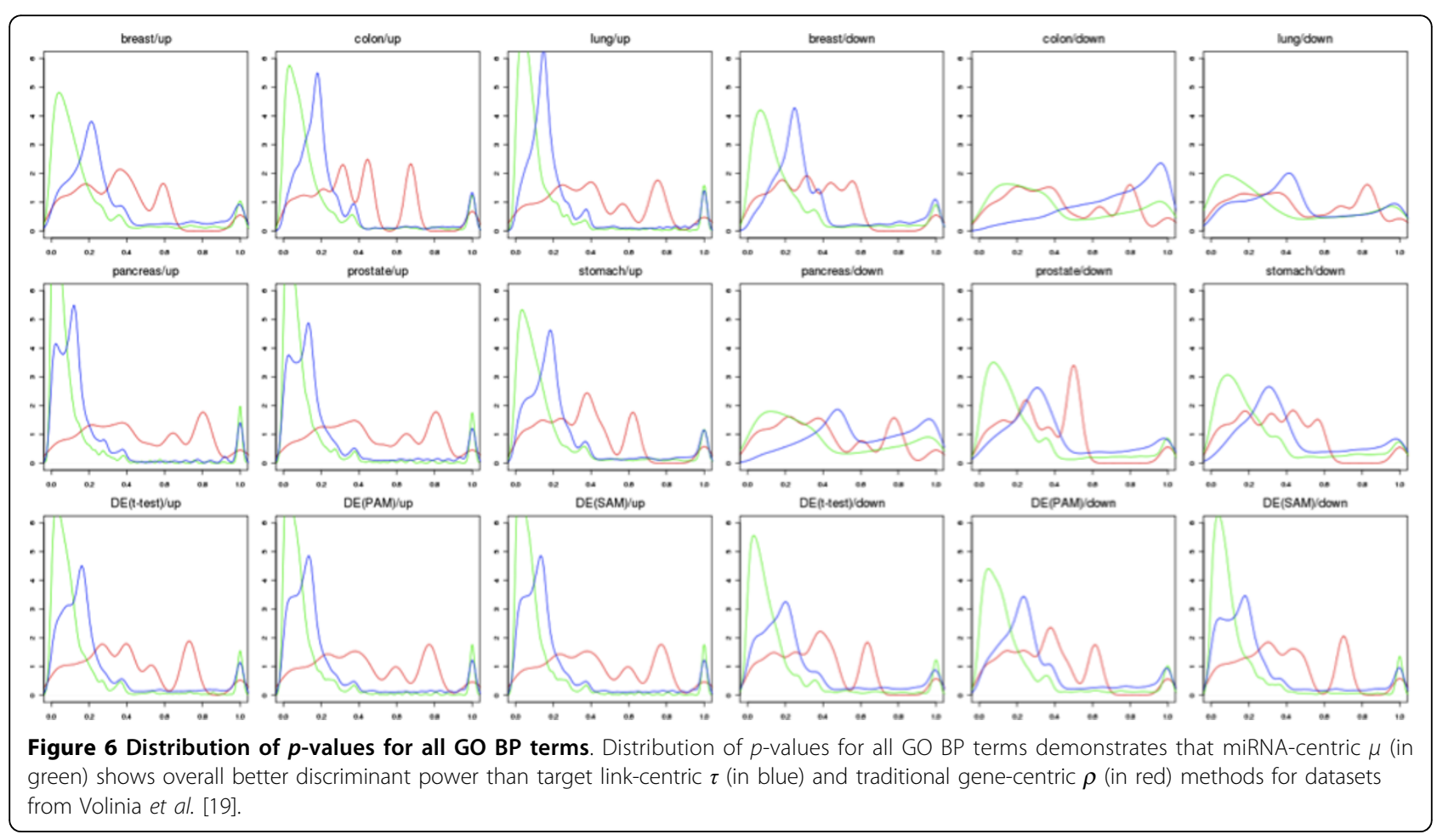


Table 2 Comparison of miRNA-centric $\mu$ and gene-centric $\rho$ measures ${ }^{\mathrm{a}}$

\begin{tabular}{|c|c|c|c|c|c|}
\hline GO terms & $\rho_{i}$ & $\rho_{j}$ & $\rho_{k}$ & $\rho_{l}$ & $p$-value ${ }_{\rho}$ (rank) \\
\hline & $\mu_{i}$ & $\mu_{j}$ & $\mu_{k}$ & $\mu_{I}$ & $p$-value ${ }_{\mu}$ (rank) \\
\hline GO:0033137, negative regulation & 3 & 7485 & 0 & 10148 & $0.0765(299)$ \\
\hline of peptidyl-serine phosphorylation & 6 & 0 & 282 & 265 & $0.0195(14)$ \\
\hline GO:0006474, N-terminal protein & 3 & 7485 & 0 & 10148 & $0.0765(299)$ \\
\hline amino acid acetylation & 6 & 0 & 320 & 227 & $0.0412(28)$ \\
\hline GO:0008054, cyclin catabolic & 2 & 7486 & 2 & 10146 & $0.5668(1842)$ \\
\hline process & 6 & 0 & 322 & 225 & $0.0427(32)$ \\
\hline \multirow[t]{2}{*}{ GO:0031047, gene silencing by RNA } & 19 & 7460 & 6 & 10142 & $0.0006(25)$ \\
\hline & 6 & 0 & 537 & 10 & $0.8958(2566)$ \\
\hline \multirow[t]{2}{*}{ GO:0030335, positive regulation of cell migration } & 34 & 7454 & 16 & 10132 & $2.31 \mathrm{e}-04(18)$ \\
\hline & 6 & 0 & 545 & 2 & $0.9783(2879)$ \\
\hline GO:0045944, positive regulation of & 150 & 7338 & 112 & 10036 & $8.62 \mathrm{e}-07(4)$ \\
\hline transcription from RNA polymerase II promoter & 6 & 0 & 546 & 1 & $0.9891(2923)$ \\
\hline GO:0006956, complement & 1 & 7487 & 0 & 10148 & $0.4246(1251)$ \\
\hline activation & 2 & 4 & 3 & 544 & $0.0010(1)$ \\
\hline GO:0060022, hard palate & 1 & 7487 & 0 & 10148 & $0.4246(1251)$ \\
\hline development & 4 & 2 & 85 & 462 & $0.0073(3)$ \\
\hline GO:0060123, regulation of growth & 1 & 7487 & 0 & 10148 & $0.4246(1251)$ \\
\hline hormone secretion & 4 & 2 & 87 & 460 & $0.0079(4)$ \\
\hline GO:0032926, negative regulation & 3 & 7485 & 0 & 10148 & $0.07652(286)$ \\
\hline of activin receptor signaling pathway & 5 & 1 & 226 & 321 & $0.0437(35)$ \\
\hline GO:0015936, coenzyme A & 1 & 7487 & 1 & 10147 & $0.6689(2086)$ \\
\hline metabolic process & 5 & 1 & 160 & 387 & $0.0102(5)$ \\
\hline
\end{tabular}

${ }^{a}$ The 'breast/up-regulated' miRNA cluster data from Volinia et al. (2006) using the target variation \#6 (see Table 1) was used.

facilitating activin signaling either by Cripto silencing or FLRG silencing inhibits human breast cancer cell growth [34,35]. Numerous studies have reported that acetyl-CoA carboxylase (ACC $\alpha$ ) and fatty acid synthase (FAS), key limiting fatty acid synthesis enzymes involved in coenzyme A metabolic process (GO:0015936), are highly expressed in human breast cancer cell lines and breast carcinomas [36-40]. Moreover, pantothenate kinase 3 (PANK3) and Coenzyme A synthase (COASY) are known breast cancer genes.

\section{Discussion}

We proposed miRNA-centric $\mu$ and target link-centric $\tau$ measures that improve functional enrichment analysis of differentially expressed or co-expressed miRNA clusters. We performed comprehensive evaluations of different methods on various settings. It is demonstrated that these new measures complement the conventional target genecentric $\rho$ measure and miRNA-centric $\mu$ method was among the most powerful and reliable.

MicroRNA's intrinsic properties of multiplicity and cooperativity [17] may be correctly modeled by combined hypergeometric distributions. Average IC value for the $\mu$ category was consistently the highest among different conditions and measures. It is suggested that the number of miRNAs and their relations associated with a specific GO term of interest is as much important as the number of target mRNAs associated with the GO term. Therefore, applying $\rho, \tau$, and $\mu$ hypergeometric distributions for functional annotation of miRNAs are mutually complementary.

The proposed method is based on computationally predicted rather than experimentally validated target relations. Computational prediction has limitations given high level of false positives and negatives. Especially, it is difficult to obtain predicted targets for minor forms of miRNA such as star, $-3 p,-5 p$ or other recently identified forms of miRNAs. All current computational enrichment analysis methods that use predicted target relations suffer from the same drawback. Combining the proposed three methods may complement with each other in finding and evaluating the correct miRNA-mRNA target relations, and improving functional annotations and enrichment analysis.

\section{Additional material}

Additional file 1: Supplementary Figures and Tables. This file

contains additional figures and tables mentioned in the main text.

\section{Acknowledgements}

This research was supported by Basic Science Research Program through the National Research Foundation of Korea (NRF) funded by the Ministry of Education, Science and Technology (2010-0028631). YRP's education grant 
was supported by the Ministry of Health and Welfare, Republic of Korea (A112020).

This article has been published as part of BMC Genomics Volume 13 Supplement 7, 2012: Eleventh International Conference on Bioinformatics (InCoB2012): Computational Biology. The full contents of the supplement are available online at http://genomebiology.com/content/supplementary/gb2010-11-1-r6-s2.xls.

\section{Author details}

${ }^{1}$ Seoul National University Biomedical Informatics (SNUBI) and Systems Biomedical Informatics Research Center, Div. of Biomedical Informatics, Seoul National University College of Medicine, Seoul 110799, Korea. ${ }^{2}$ Institute of Endemic Diseases, Medical Research Center, Seoul National University, Seoul 110799, Korea.

\section{Authors' contributions}

$S L$ and JK conceived and designed the study. SL performed the experiments. KS and JK helped to refine the analysis and the interpretation of results. SL, KS, and JK wrote the manuscript.

\section{Competing interests}

The authors declare that they have no competing interests.

Published: 13 December 2012

\section{References}

1. Nelson PT, Baldwin DA, Scearce LM, Oberholtzer JC, Tobias JW, Mourelatos Z: Microarray-based, high-throughput gene expression profiling of microRNAs. Nat Methods 2004, 1(2):155-161.

2. Lai EC: microRNAs: runts of the genome assert themselves. Curr Biol 2003, 13(23):R925-936

3. Ambros V: The functions of animal microRNAs. Nature 2004 431(7006):350-355.

4. Bartel DP: MicroRNAs: genomics, biogenesis, mechanism, and function. Cell 2004, 116(2):281-297.

5. Lim LP, Lau NC, Garrett-Engele P, Grimson A, Schelter JM, Castle J, Bartel DP, Linsley PS, Johnson JM: Microarray analysis shows that some microRNAs downregulate large numbers of target mRNAs. Nature 2005, 433(7027):769-773

6. Ulitsky I, Laurent LC, Shamir R: Towards computational prediction of microRNA function and activity. Nucleic Acids Res 2010, 38(15):e160.

7. Wu YQ, Chen DJ, He HB, Chen DS, Chen LL, Chen HC, Liu ZF: Pseudorabies virus infected porcine epithelial cell line generates a diverse set of host microRNAs and a special cluster of viral microRNAs. PloS one 2012, 7(1): e30988.

8. Xiao $Y, X u$ C, Guan J, Ping Y, Fan $H, L i Y$, Zhao H, Li X: Discovering dysfunction of multiple microRNAs cooperation in disease by a conserved microRNA co-expression network. PloS one 2012, 7(2):e32201.

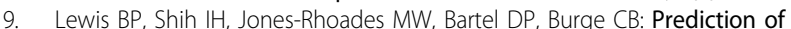
mammalian microRNA targets. Cell 2003, 115(7):787-798.

10. Grimson A, Farh KK, Johnston WK, Garrett-Engele P, Lim LP, Bartel DP. MicroRNA targeting specificity in mammals: determinants beyond seed pairing. Mol Cell 2007, 27(1):91-105.

11. Krek A, Grun D, Poy MN, Wolf R, Rosenberg L, Epstein EJ, MacMenamin P, da Piedade I, Gunsalus KC, Stoffel M, et al: Combinatorial microRNA target predictions. Nat Genet 2005, 37(5):495-500.

12. Gaidatzis D, van Nimwegen E, Hausser J, Zavolan M: Inference of miRNA targets using evolutionary conservation and pathway analysis. $B m C$ Bioinformatics 2007, 8:69

13. Gusev Y: Computational methods for analysis of cellular functions and pathways collectively targeted by differentially expressed microRNA. Methods 2008, 44(1):61-72.

14. $\mathrm{Xu}$ J, Wong C: A computational screen for mouse signaling pathways targeted by microRNA clusters. RNA 2008, 14(7):1276-1283.

15. Nam S, Kim B, Shin S, Lee S: miRGator: an integrated system for functional annotation of microRNAs. Nucleic Acids Res 2008, 36(Database issue):D159-164

16. Creighton CJ, Nagaraja AK, Hanash SM, Matzuk MM, Gunaratne PH: A bioinformatics tool for linking gene expression profiling results with public databases of microRNA target predictions. RNA 2008, 14(11):2290-2296.
17. John B, Enright AJ, Aravin A, Tuschl T, Sander C, Marks DS: Human microRNA targets. PLoS Biol 2004, 2(11):e363.

18. Ruepp A, Kowarsch A, Schmidl D, Buggenthin F, Brauner B, Dunger I, Fobo G, Frishman G, Montrone C, Theis FJ: PhenomiR: a knowledgebase for microRNA expression in diseases and biological processes. Genome Biol 2010, 11(1):R6

19. Volinia S, Calin GA, Liu CG, Ambs S, Cimmino A, Petrocca F, Visone R, lorio $M$, Roldo $C$, Ferracin $M$, et al: A microRNA expression signature of human solid tumors defines cancer gene targets. Proc Natl Acad Sci USA 2006, 103(7):2257-2261.

20. Hon LS, Zhang Z: The roles of binding site arrangement and combinatorial targeting in microRNA repression of gene expression. Genome Biol 2007, 8(8):R166.

21. Sethupathy P, Megraw M, Hatzigeorgiou AG: A guide through present computational approaches for the identification of mammalian microRNA targets. Nat Methods 2006, 3(11):881-886.

22. Xiao F, Zuo Z, Cai G, Kang S, Gao X, Li T: miRecords: an integrated resource for microRNA-target interactions. Nucleic Acids Res 2009, 37(Database issue):D105-110

23. Megraw M, Sethupathy $P$, Corda B, Hatzigeorgiou AG: miRGen: a database for the study of animal microRNA genomic organization and function. Nucleic Acids Res 2007, 35(Database issue):D149-155.

24. Elston RC: On Fisher's method of combining p-values. Biometrical Journal 1991, 33:339-345.

25. Kugler KG, Mueller LA, Graber A: MADAM - An open source meta-analysis toolbox for R and Bioconductor. Source code for biology and medicine 2010, 5:3.

26. Lord PW, Stevens RD, Brass A, Goble CA: Investigating semantic similarity measures across the Gene Ontology: the relationship between sequence and annotation. Bioinformatics 2003, 19(10):1275-1283.

27. Ozer HG, Chen J, Zhang F, Yuan B: Clustering of eukaryotic orthologs based on sequence and domain similarities using the Markov graphflow algorithm. 2004 [http://www.biosci.ohio-state.edu/ ozer/pub/papers/ icba04 hg ozer.pdf].

28. Resnik P: Using information content to evaluate semantic similarity in a taxonomy. Proceedings of the 14th International Joint Conference on Artificial Intelligence 1995, 1:448-453.

29. Markiewski MM, Lambris JD: Is complement good or bad for cancer patients? A new perspective on an old dilemma. Trends Immunol 2009, 30(6):286-292

30. Niculescu F, Rus HG, Retegan M, Vlaicu R: Persistent complement activation on tumor cells in breast cancer. The American journal of pathology 1992, 140(5):1039-1043.

31. Privat M, Aubel C, Arnould S, Communal Y, Ferrara M, Bignon YJ: Breast cancer cell response to genistein is conditioned by BRCA1 mutations. Biochem Biophys Res Commun 2009, 379(3):785-789.

32. Cassoni P, Papotti M, Ghe C, Catapano F, Sapino A, Graziani A, Deghenghi R, Reissmann T, Ghigo E, Muccioli G: Identification, characterization, and biological activity of specific receptors for natural (ghrelin) and synthetic growth hormone secretagogues and analogs in human breast carcinomas and cell lines. J Clin Endocrinol Metab 2001, 86(4):1738-1745.

33. Hankinson SE, Willett WC, Colditz GA, Hunter DJ, Michaud DS, Deroo B, Rosner B, Speizer FE, Pollak M: Circulating concentrations of insulin-like growth factor-I and risk of breast cancer. Lancet 1998, 351(9113):1393-1396.

34. Adkins HB, Bianco C, Schiffer SG, Rayhorn P, Zafari M, Cheung AE, Orozco O, Olson D, De Luca A, Chen LL, et al: Antibody blockade of the Cripto CFC domain suppresses tumor cell growth in vivo. J Clin Invest 2003, 112(4):575-587.

35. Razanajaona D, Joguet S, Ay AS, Treilleux I, Goddard-Leon S, Bartholin L, Rimokh R: Silencing of FLRG, an antagonist of activin, inhibits human breast tumor cell growth. Cancer Res 2007, 67(15):7223-7229.

36. Alo PL, Visca P, Trombetta G, Mangoni A, Lenti L, Monaco S, Botti C, Serpieri DE, Di Tondo U: Fatty acid synthase (FAS) predictive strength in poorly differentiated early breast carcinomas. Tumori 1999, 85(1):35-40.

37. Milgraum LZ, Witters LA, Pasternack GR, Kuhajda FP: Enzymes of the fatty acid synthesis pathway are highly expressed in in situ breast carcinoma. Clinical cancer research: an official journal of the American Association for Cancer Research 1997, 3(11):2115-2120. 
38. Nakamura I, Kimijima I, Zhang GJ, Onogi H, Endo Y, Suzuki S, Tuchiya A, Takenoshita S, Kusakabe T, Suzuki T: Fatty acid synthase expression in Japanese breast carcinoma patients. International journal of molecular medicine 1999, 4(4):381-387.

39. Sinilnikova OM, Ginolhac SM, Magnard C, Leone M, Anczukow O, Hughes D, Moreau K, Thompson D, Coutanson C, Hall J, et al: Acetyl-CoA carboxylase alpha gene and breast cancer susceptibility. Carcinogenesis 2004, 25(12):2417-2424.

40. Witters LA, Widmer J, King AN, Fassihi K, Kuhajda F: Identification of human acetyl-CoA carboxylase isozymes in tissue and in breast cancer cells. The International journal of biochemistry 1994, 26(4):589-594.

doi:10.1186/1471-2164-13-S7-S17

Cite this article as: Lee et al.: MicroRNA-centric measurement improves

functional enrichment analysis of co-expressed and differentially expressed microRNA clusters. BMC Genomics 2012 13(Suppl 7):S17.

\section{Submit your next manuscript to BioMed Central} and take full advantage of:

- Convenient online submission

- Thorough peer review

- No space constraints or color figure charges

- Immediate publication on acceptance

- Inclusion in PubMed, CAS, Scopus and Google Scholar

- Research which is freely available for redistribution

Submit your manuscript at www.biomedcentral.com/submit
() Biomed Central 